\title{
A Pricing Model for American Options with Stochastic Interest Rates
}

\author{
Bert Menkveld* and Ton Vorst
}

Department of Finance

Erasmus University Rotterdam

P.O. Box 1738

NL-3000 DR Rotterdam

The Netherlands

e-mail: vorst@few.eur.nl

Tel. 31-10-4081270; Fax: 31-10-4527746

April 20, 1998

*Bert Menkveld, Department of Finance, Erasmus University, e-mail: menkveld@few.eur.nl and Investor Relations, KLM Royal Dutch Airlines 


\section{A Pricing Model for American Options with Stochas-}

\section{tic Interest Rates ${ }^{1}$}

\section{Abstract}

In this paper we introduce a new methodology to price American put options under stochastic interest rates. We construct a binomial tree for the forward risk adjusted process and calculate analytically the expected early exercise value in each point. For American puts the correlation between the stock price and the interest rate has different influences on European option values and early exercise premiums. This results in a nonmonotonic relation between this correlation and the American put value. Furthermore, there is evidence that the early exercise premium due to stochastic interest rates is much larger than established by other researchers.

\footnotetext{
${ }^{1}$ Acknowledgement: We are thankful to San-Lin Chung, Antoon Pelsser and participants at the Global Derivatives Meeting in Paris, the APMOD-meeting in Limassol and seminars at University of Groningen and Erasmus University Rotterdam for helpful comments on earlier versions of this paper. The usual disclaimer applies.
} 


\section{A Pricing Model for American Options with Stochastic In- terest Rates}

\section{Introduction}

American stock option valuation has been an important research subject over the last two decades. However, in most research it is assumed that interest rates are constant. Recently, Amin and Bodurtha (1995), Ho, Stapleton and Subrahmanyan (1997) and Chung (1997) have considered pricing of American options with stochastic interest rates.

In this paper we develop a new method to value American stock options with stochastic interest rates. In stead of a multivariate binomial tree as in Ho et al. (1997) we construct a simple binomial tree for the stock price divided by the price of the zero coupon bond that matures at the maturity date of the option. In fact, we construct a tree for the so-called forward risk adjusted measure. In each node of the tree the quotient of the stock price and bond price is constant and there are combinations of stock and bond prices for which immediate exercise is optimal and other combinations for which this is not the case. We derive for each node in the tree an analytic expression for the expected immediate exercise premium conditional on this quotient of stock and bond prices. This immediate exercise premium is added to the value that is derived from the familiar backward procedure. 
For our method we only need a simple binomial tree in contrast to the multivariate tree of Ho et al. (1997). Also Chung (1997) uses the forward risk adjusted measure, but just as Ho et al. (1997) he considers options that are only exercisable at two fixed points in time. Then values of options are derived by applying the backward procedure to the forward risk adjusted tree. Next Ho et al. (1997) apply Richardson extrapolation to approximate the value of the American option under stochastic interest rates. We allow for early exercise at each point of our tree and do not use Richardson extrapolation. In fact, our more refined trees show that prices derived by Richardson extrapolation significantly underestimate the true American put option value.

If Ho et al. (1997) want to refine their Richardson extrapolation and consider the possibility of exercising at three fixed points in time, they would need a multivariate tree with six underlying variables. As stated before, our methodology uses only one tree and at the same time allows for early exercise in each point of the tree.

Of course, both European and American option prices depend among others on the correlation between the interest rate process and the stock price process. It is interesting to see that with increasing correlation between the interest rate process and the stock price process, and hence a decreasing correlation between bond and stock prices, the values of European options increase, while the values of the early exercise premium decrease. For American options this might result in a non-monotonic relation between the correlation coefficient and the option 
price.

Especially, for long term options it is not always clear which interest rate should be used in a nonstochastic interest rate option pricing model. Our model allows for the incorporation of a full term structure model. Hence, it does not only justice to the stochastic nature of interest rates, but also takes both short and long term rates into account in an appropriate way.

This paper is organized as follows. In the next section we explain our basic methodology for pricing American options with stochastic interest rates. In section 2 we give numerical illustrations of our methodology. We look at convergence issues, the influence of the stochastic interest rates and the correlation between stock and bond price processes. Furthermore, we compare our results with those of Ho et al. (1997) and of Chung (1997) and consider the influence of the shape of the term structure on option prices. Section 3 concludes the paper.

\section{The pricing model for American options}

In this section we focus on the pricing of American put options on stocks that do not pay dividends in a stochastic interest rate environment. For the stock price we specify the familiar geometric Brownian motion

$$
d S(t)=\alpha S(t) d t+\sigma_{1} S(t) d W_{1}(t)
$$


while we use the Hull-White (extended-Vasicek) model (1994) to specify the short term interest rate process

$$
d r(t)=[\theta(t)-\operatorname{ar}(t)] d t+\sigma_{2} d W_{2}(t) .
$$

The coefficient of correlation between $d W_{1}(t)$ and $d W_{2}(t)$ is assumed to be equal to a constant $\rho$. Usually $\rho$ will be negative since increasing interest rates have a depressing effect on stock prices. In the rest of the paper we will sometimes suppress in our notation the time dependence of $S, r, W_{1}$ and $W_{2}$.

We assume that the put option matures at time $T$, has an exercise price $K$ and $B(t, T)$ is the price at time $t$ of a discount bond that pays one unit at time $T$.

Let $P(S(t), B(t, T))$ be the price of the American put option. We use the bond price $B(t, T)$ as a numeriaire and have the following notation

$$
\begin{gathered}
\tilde{S}(t)=S(t) / B(t, T) \\
\tilde{P}(S(t), B(t, T))=P(S(t), B(t, T)) / B(t, T)
\end{gathered}
$$

If there are no arbitrage opportunities there exists an equivalent measure such that $\tilde{S}$ and $\tilde{P}$ are martingales. This martingale measure is called the T-forward risk adjusted measure as in Jamshidian (1991) and Musiela and Rutkowski (1997, chapter 13). Expectations and variances under this measure are denoted by $E^{*}$ and $V a r^{*}$. To value the American put option we first build a recombining 
binomial tree under the T-forward risk adjusted measure for $\tilde{S}(t)$. Since the volatility of $S(t)$ is constant and that of $B(t, T)$ is decreasing over time, the volatility of $\tilde{S}(t)$ is not constant and we cannot use a standard binomial tree. In appendix 1 a tree is constructed where the time intervals between two nodes in the tree vary over time. To calculate the American put price we use the familiar backward procedure but in each node of the tree correct for the early exercise value as illustrated next. Consider the following typical triangles in the tree

$$
\begin{aligned}
& \tilde{S}(t) u \\
& \tilde{S}(t) \\
& \tilde{S}(t) d
\end{aligned}
$$

for the stock price divided by the numeriaire $B(t, T)$ and

$$
\begin{array}{cc} 
& \tilde{P}(\tilde{S}(t) u) \\
& \tilde{P}(\tilde{S}(t)) d t)
\end{array}
$$

for the put option price again divided by the numeraire $B(t, T)$. Let $p$ be the T-forward risk adjusted probability of an upward move.

$$
\tilde{P}(\tilde{S}(t))=p \tilde{P}(\tilde{S}(t) u)+(1-p) \tilde{P}(\tilde{S}(t) d)+E E V^{*} \triangleq \tilde{P}_{0}(\tilde{S}(t))+E E V^{*}
$$

where $E E V$ is the early exercise value, which still has to be determined. In each node of the tree, such as $\tilde{S}(t)$, the value of $S(t) / B(t, T)$ is constant, say $\bar{c}$, but $S(t)$ and $B(t, T)$ vary. Hence, for some low values of $S(t)$, early exercise at 
that node might be optimal, while this is not the case for other values at this same node. Since, we have to calculate the expected early exercise value over all possible price processes and have all prices discounted in the arbitrage free economy, we have

$$
E E V^{*}=E_{\bar{c}}^{*} \max \left(\frac{K-S(t)}{B(t, T)}-\tilde{P}_{0}(\tilde{S}(t)), 0\right)
$$

where $E_{\bar{c}}^{*}$ is the conditional expectation under $S(t) / B(t, T)=\bar{c}$. Hence

$$
E E V^{*}=E_{\bar{c}}^{*} \max \left(\frac{K}{B(t, T)}-\left(\bar{c}+\tilde{P}_{0}(\tilde{S}(t))\right), 0\right)
$$

Since, in this formula $\bar{c}, K$ and $\tilde{P}_{0}(S(t))$ are constants, an analytic expression for $E E V^{*}$ can be found if we know the conditional distribution of $B(t, T)$ under the $T$-forward risk adjusted measure.

Under the $T$-forward risk adjusted measure both $S(t) / B(t, T)$ and $B(t, s) / B(t, T)$, for $t<s$ are martingale processes. Hence,

$$
d \tilde{S}(t)=\sigma_{\tilde{s}}(t, T) \tilde{S}(t) d W_{1}^{*}(t)
$$

and

$$
d \tilde{B}(t, s)=\sigma_{\tilde{B}}(t, s) \tilde{B}(t, s) d W_{2}^{*}(t),
$$

where $\sigma_{\tilde{s}}(t, s)$ and $\sigma_{\tilde{B}}(t, s)$ are determined in appendix 1 , and $W_{1}^{*}(t)$ and $W_{2}^{*}(t)$ are Brownian motions. From the martingale property it follows that:

$$
E^{*}\left(\frac{S(t)}{B(t, T)}\right)=\frac{S(0)}{B(0, T)}
$$


and

$$
E^{*}\left(\frac{1}{B(t, T)}\right)=E^{*}\left(\frac{B(t, t)}{B(t, T)}\right)=\frac{B(0, t)}{B(0, T)}
$$

Furthermore, it follows from (8) and (9) that $\ln \tilde{S}(t)$ and $\ln \tilde{B}(t, s)$ are bivariate normally distributed with:

$$
\begin{gathered}
\operatorname{Var}^{*}\left(\ln \left(\frac{S(t)}{B(t, T)}\right)\right)=\int_{0}^{t} \sigma_{\tilde{s}}(\tau, T)^{2} d \tau \\
\operatorname{Var}^{*}\left(\ln \left(\frac{1}{B(t, T)}\right)\right)=\operatorname{Var}^{*}\left(\ln \left(\frac{B(t, t)}{B(t, T)}\right)\right)=\int_{0}^{t} \sigma_{\tilde{B}}(\tau, t)^{2} d \tau
\end{gathered}
$$

Explicit formulas for these expressions can be calculated, given the specifications of $\sigma_{\tilde{s}}^{2}$ and $\sigma_{\tilde{B}}^{2}$ in appendix 1 , where also is indicated how an explicit expression for $C o v^{*}(\ln \tilde{S}(t), \ln B(t, T))$ can be derived.

Now (10) and (11) imply that

$$
E^{*}(\ln S(t) / B(t, T))=\ln (S(0) / B(0, T))-\frac{1}{2} \operatorname{Var}^{*} \ln (S(t) / B(t, T))
$$

and we find a similar expression for $E^{*} \ln (1 / B(t, T))$.

Since $\ln (S(t) / B(t, T))$ and $\ln (1 / B(t, T))$ are bivariate normally distributed we know that the conditional distribution of $\ln (1 / B(t, T))$ given that $S(t) / B(t, T)=$ $\bar{c}$ is normal and the mean and variance can be explicitly calculated. This allows us to give an analytic expression for $E E V^{*}$ in equation (7). The details are given in appendix 2. Hence, we can calculate American put option prices by the familiar backward procedure and the adjustment (7).

Of course, we can also use this binomial tree to calculate the value of European options by simply ignoring the early exercise value $E E V^{*}$. Also analytic 
expressions for European options with stochastic interest rates can be derived by standard procedures based on the $T$-forward risk measure. If $p$ denotes the price of a European put option we have

$$
\begin{aligned}
p(S(0), B(0, T)) & =B(0, T) \tilde{p}(S(0) / B(0, T)) \\
& =B(0, T) E^{*} \max (K-S(T) / B(T, T), 0)
\end{aligned}
$$

Under the $T$-forward risk-adjusted process $\ln (S(T) / B(T, T))$ is normally distributed with $E^{*}(S(T) / B(T, T))=S(0) / B(0, T)$ and $\operatorname{Var}^{*}(\ln (S(T) / B(T, T)))=$

$\int_{0}^{T} \sigma^{2}(\tau, T) d \tau$ as explained above. Hence, an analytic expression for (7) can be given using standard procedures.

In the next section we compare the binomial tree values of European options with their analytical counterparts to assess the convergence properties of the tree.

\section{Numerical Examples}

In this section we will illustrate our methodology with some numerical examples and it is divided in four subsections. In this first subsection we assess the convergence properties of our binomial tree. We consider both European, where we also have anlytic solutions and American options. In the second subsection we consider the influence of stochastic interest rates on the values of European and American put options and especially consider different correlations between the stock price process and the interest rate process. In subsection 3 we will compare our results with those of Ho et al. (1997) and Chung (1997). In these 
three subsections we assume that the initial term structure is flat. In the final subsection we relax this assumption by looking at upward sloping and downward sloping initial term structures.

\subsection{Convergence properties}

Figure 1 shows the dependence of the binomial tree values of European and American put options on the number of steps in the tree. All options have a maturity of one year, the term structure of interest rates is flat at $7 \%$. The strike price and initial stock price are 1 . The mean reversion parameter $a$ and the volatility $\sigma_{2}$ of the interest rate process are respectively 0.1 and 0.01 as in Hull and White (1994). In figures $1 \mathrm{a}, \mathrm{b}$ and $\mathrm{c}$ the volatility of the underlying stock is $20 \%$, while the coefficient of correlation $\rho$ between the stock price process and the interest rate process is given by $-0,5 ; 0$ and 0,5 respectively. In figure $1 \mathrm{~d}$ we have set the volatility of the underlying stock at $40 \%$ and a zero correlation. We see the familiar zigzag pattern for binomial trees if we increase the number of

steps. However, in general the convergence of our method is good, while there is also a clear convergence to the analytical price for European options.

\subsection{The influence of stochastic interest rates}

In table 1 European and American put option prices are given for different values of the interest rate and the volatility of the underlying stock, where we vary the 
coefficient of correlation among the rows of the table. EEV stands for the early exercise premium and is the difference between the American put option price $P$ and the European put option price p. In the nonstochastic interest rate case $(N S)$ we have set $\sigma_{2}=0$. In all other cases $\sigma_{2}=0.01$ and the mean reversion parameter $a=0.1$. All prices are calculated with binomial trees with 100 steps.

Most interesting is the influence of the correlation between the interest rate process and the stock price. If the correlation increases, and hence the correlation between bond and stock prices decreases, the European option price increases while the early exercise value decreases. This results in an American option value that is no longer monotonic as a function of the correlation coefficient. This is also illustrated for some specific cases in figures 2.1 and 2.2. If the correlation between interest rates and stock prices increases, the correlation between bond prices and stock prices decreases and the volatility of the quotient of stock and bond prices increases. Hence, in the forward risk adjusted measure the volatility of this quotient which determines the European option price is higher and thus the European option price is higher. However, a lower correlation means that low stock prices come with higher interest rates, which makes it more attractive to early exercise put options. Hence, the influence of the correlation coefficient differs for the European option value and the early exercise value. 


\subsection{Comparison with other methods}

Also Ho et al. (1997) and Chung (1997) have calculated American put option values with stochastic interest rates. In both papers Richardson extrapolation is used based on values of an option that is only exercisable at maturity $T$, i.e. a European option and an option that is exercisable at maturity or half way, $(T / 2)$ between the actual date and the maturity date. Therefore, these authors do not specify a full term structure model, but only the volatility of the price at time $T / 2$ of a bond that matures at time $T$ and the correlation of this bond with the stock price. Hence, in order to compare our methodology with the above mentioned ones, we have to set the volatility of the interest rate process

$\sigma_{2}$, the mean reversion rate $a$ and the correlation $\rho$ such that these imply the same volatility and correlation as in the other papers for the price at time $T / 2$ of a bond that matures at time $T$. The variances and covariances are explicitly given in appendix 1 and from these $\sigma_{2}$ and $\rho$ can be readily inferred such that as in Ho et al. (1997) and Chung (1997) we have $\operatorname{Var}(\ln (B(T / 2), T))=.02$ and $\rho$ $=-0.3$. Furthermore, we also adjust $\sigma_{1}$ such that the European option values do not change due to the introduction of stochastic interest rates.

In table 2 we compare our methodology with that of Ho et al. (1997) and Chung (1997). For the nonstochastic case we can also compare with the prices derived by Geske and Johnson (1984). The parameter values for $r$ and $\sigma$ are taken from Geske and Johnson (1984) while the American put option prices 
in the different columns are taken from the earlier mentioned papers. In the nonstochastic case all our prices are closer or at least as close to the GeskeJohnson prices than the prices of Ho et al. and Chung. Hence, we can conclude that our method is very accurate in the nonstochastic case. Of course, it is more interesting to study the prices with stochastic interest rates. The last column of table 2 shows that our prices are significantly higher than those of Ho et al. and Chung. Since our method allows for early exercise at each moment in time and the two others only at option maturity and half way, we also applied our methodology where we only allow for exercising at these particular points in time and then applied Richardson extrapolation. The results are given in column (10) under $P_{S R}^{M V 2}$. The differences with the other methods are reasonably small, but since we use a full term structure model and Ho et al. (1997) and Chung (1997) only specify the variance of the price of a particular discount bond over a specific time interval, we will never get exactly the same prices in $P_{S R}^{M V 2}$ as for the other methods. Hence, the higher values in column (11) are due to the extended exercise possibilities and Richardson extrapolation does not seem to work very well for American options with stochastic interest rates. The early exercise premiums seem to be very large, but this is due to the negative correlations between the interest rates and stock prices. Low stock prices come with high interest rates and hence in these cases early exercising is very profitable. In case of a positive correlation between stock prices and interest rates the early exercise premium is much smaller as shown in figures 2.1 and 2.2. However, this latter case is 
less realistic. Also the relative differences with the other methods are larger in cases of low volatilities of the stock prices, since in these cases the interest rate volatility is more important in a relative sense. We must conclude with Ho et al. (1997) that stochastic interest rates have a significant impact on the early exercise value of American put options but that it is even larger than described by these authors.

\subsection{Different shapes of the term structure}

In this subsection we consider the influence of the term structure of interest rate on the pricing of American puts. We consider three term structures, an upward sloping one with $r(t)=0.08-0.05 \exp (-0.18 t)$, a downward sloping (inverted) one with $r(t)=-0.00353+0.05 \exp (-0.18 t)$ and a flat term structure with $r(t)=0.0382$. All term stuctures considered have for the option maturity, which is one year, the same interest rate of 0.0382 . We vary the volatility $\sigma$ of the underlying stock and the correlation coefficient $\rho$ as before. The resulting prices are given in table 3. For all term structures the European prices are the same, given $\sigma$ and $\rho$, since all three term structures have the same one year interest rates. Hence, differences are only due to the early exercise feature. We see that the early exercise premium is higher for the upward sloping term structure, than for the flat term structure, which is again higher than for the downward sloping term structure. At first sight, this seems counterintuitive since all interest rates 
for the upward sloping term structure are lower and early exercising does have a higher payoff with higher interest rates. However, an upward sloping term structure implies higher forward interest rates and hence higher rates in the later part of the year during which the option runs. It is during this period that the underlying asset might have a low enough price to make early exercise profitable. Also in this table we see that for all term structures the European option value increases with increasing correlation between the stock price process and the interest rate process, while the early exercise premium decreases.

\section{Conclusion}

In this paper we introduced a new methodology to price American put options under stochastic interest rates. The method is a combination of an analytic approach and a binomial tree approach. We have constructed a binomial tree for the forward risk adjusted tree and calculate analytically the expected early exercise value in each point. The methodology might be applied in other cases too. Obviously, these include convertible bonds, since the underlying processes are again interest rate processes and stock prices, but also to other options where there are two underlying stochastic processes such as American lookback options or American average rate options. For American puts with stochastic interest rates the main conclusions from the numerical experiments are that the correlation between the stock price process and the interest rate process has different 
influences on the European option values and the early exercise premiums. This results in a nonmonotonic relation between this correlation and the American put option value. Furthermore, there is evidence that the early exercise premium due to stochastic interest rates is much larger than established before by other researchers.

\section{References}

[1] Amin, K.I. and J.N. Bodurtha (1995). Discrete-Time Valuation of American Options with Stochastic Interest Rates, The Review of Financial Studies 8 (1), 193-234.

[2] Chung, S. (1997). American Option Valuation under Stochastic Interest Rates, paper presented at the 24th EFA Meeting, Vienna.

[3] Geske, R. and H. Johnson (1984). The American Put Option Valued Analytically, Journal of Finance 39 (5), 1511-1524.

[4] Ho, T.S., R.C. Stapleton and M.G. Subrahmanyan (1997), The Valuation of American Options with Stochastic Interest Rates: A Generalization of the Geske-Johnson Technique, Journal of Finance 52, 827-840.

[5] Hull, J. and A. White (1994). Numerical Processes for Implementing Term Structure Models I: Single Factor Models, Journal of Derivatives, Fall, 7-16. 
[6] Jamshidian, F. (1991). Bond and Option Evaluation in the Gaussian Interest Rate Model, Research in Finance 9, 131-170.

[7] Musiela, M. and M. Rutkowski (1997). Martingale Methods in Financial Modelling, Springer Verlag, Berlin Heidelberg New York.

\section{Appendix 1}

In this appendix we construct a binomial tree for $\tilde{S}(t)=S(t) / B(t, T)$ and specify the joint distribution of $\ln (\tilde{S}(t))$ and $1 / \ln (B(t, T))$ under the forward risk adjusted measure.

Given the instantaneous short term process $r(t)$ as in equation (2) it follows from among others Hull and White (1994) that

$$
B(t, T)=H(t, T) e^{-G(t, T) r(t)}
$$

with $G(t, T)=\frac{1}{a}\left[1-e^{-a(T-t)}\right]$,

$$
\ln H(t, T)=\ln (B(0, T) / B(0, t))+G(t, T) F(0, t)-\frac{\sigma^{2}}{4 a}\left(1-e^{-2 a t}\right) G(t, T)^{2}
$$

where $F(0, t)=-\frac{\partial}{\partial t} \ln B(0, t)$ is the instantaneous forward rate and $\theta(t)=$ $F_{t}(0, t)+a F(0, t)+\frac{\sigma^{2}}{2 a}\left(1-e^{-2 a t}\right)$.

It follows from Ito's lemma that

$$
\begin{array}{r}
d B(t, T)=B_{r}(t, T) d r+\frac{1}{2} \sigma_{2}^{2} B_{r r}(t, T) d t+B_{t}(t, T) d t= \\
K(t, T, r) d t-G(t, T) B(t, T) \sigma_{2} d W_{2}(t),
\end{array}
$$


where $B_{r}(t, T), B_{r r}(t, T)$ and $B_{t}(t, T)$ are partial derivatives and $K(t, T, r)$ is the drift.

Hence $\sigma_{\tilde{s}}(t, T)$ in the martingale process (8) is given by:

$$
\sigma_{\tilde{s}}^{2}(t, T)=\left[\sigma_{1}^{2}+G(t, T)^{2} \sigma_{2}^{2}+2 \rho \sigma_{1} \sigma_{2} G(t, T)\right]
$$

thus $\operatorname{var}^{*}(\ln (\tilde{S}(t)))=\int_{0}^{t}\left(\sigma_{1}^{2}+\sigma_{2}^{2} G(\tau, T)^{2}+2 \rho \sigma_{1} \sigma_{2} G(\tau, T)\right) d \tau$.

Similarly $\sigma_{\tilde{B}}(t, s)$ in $(9)$ is given by

$$
\sigma_{\tilde{B}}(t, s)=\sigma_{2}(G(t, s)-G(t, T))
$$

and thus $\operatorname{var}^{*}(\ln (B(t, T)))=\frac{\sigma_{2}^{2}}{2 a}\left(1-e^{2 a t}\right) G(t, T)^{2}$.

Furthermore

$$
\begin{gathered}
\operatorname{Cov}^{*}(\ln \tilde{S}(t), \ln B(t, T))=-\operatorname{Cov}^{*}(\ln \tilde{S}(t), \ln \tilde{B}(t, t))= \\
\int_{0}^{t} \rho \sigma_{1} \sigma_{2}[G(\tau, t)-G(\tau, T)]+\sigma_{2}^{2}\left[G(\tau, t) G(\tau, T)-G(\tau, T)^{2}\right] d \tau
\end{gathered}
$$

It follows from (18) that the volatility of the process of $\tilde{S}(t)$ is not constant. To construct a recombining binomial tree we do not divide the interval $[0, T]$ in periods of equal length, but in periods of equal volatility. We chose time points $0=t_{0}<t_{1}<t_{2} \ldots<T_{n}=T$ such that $\int_{t_{i-1}}^{t_{i}} \sigma_{\tilde{s}}^{2}(t, T) d t$ is independent of $i$. 


\section{Appendix 2}

Derivation of the early exercise value $E E V$ in equation (7).

Define $a=K, b=\bar{c}+\tilde{P}_{*}(\tilde{S}(t))$, then

$$
E E V^{*}=E_{\bar{c}}^{*} \max \left(\frac{a}{B(t, T)}-b, 0\right)
$$

under the conditional distribution for $1 / B(t, T)$ given that $S(t) / B(t, T)=\bar{c}$. Let $X=\ln B(t, T)$ and $Y=\ln \frac{S(t)}{B(t, T)}$. Next we use well known formula's to find the conditional normal distribution of $X$ given that $Y=\ln \bar{c}$, i.e.

$$
m=E\left(\ln B(t, T) \mid \ln \frac{S(t)}{B(t, T)}=\ln \bar{c}\right)=E X+\frac{\sigma_{X Y}}{\sigma_{Y}^{2}}(\ln \bar{c}-E Y)
$$

and

$$
v^{2}=\operatorname{Var}\left(\ln B(T, t) \mid \ln \frac{S(t)}{B(t, T)}=\ln \bar{c}\right)=\sigma_{X}^{2}-\frac{\sigma_{X Y}^{2}}{\sigma_{Y}^{2}}
$$

Define $x$ by

$$
\left(\ln B(t, T) \mid \ln \frac{S(t)}{B(t, T)}=\ln \bar{c}\right)=m+v x
$$

where $x$ is a standard normal random variable. Then

$$
E E V^{*}=\int_{-\infty}^{f}\left(\frac{a}{e^{m+v x}}-b\right) \frac{e^{-x^{2} / 2}}{\sqrt{2 \pi}} d x
$$

with $f=[\ln (a / b)-m] / v$. By standard procedures this integral can be evaluated, which results in

$$
E E V=a e^{-m+v^{2} / 2} N(f+v)-b N(f)
$$

where $N$ is the standard normal distribution function. 
Figure 1a: Value of Put Option

$\mathrm{S}=1, \mathrm{~K}=1, \mathrm{R}=0.07$ (flat term structure),

$\sigma 1=0.20, \sigma 2=0.01, \rho(\mathrm{r}, \mathrm{S})=-0.5, \mathrm{a}=0.1$
European option value, forward rate adjusted tre

American option value,

forward rate adjusted tree

Analytic value European option

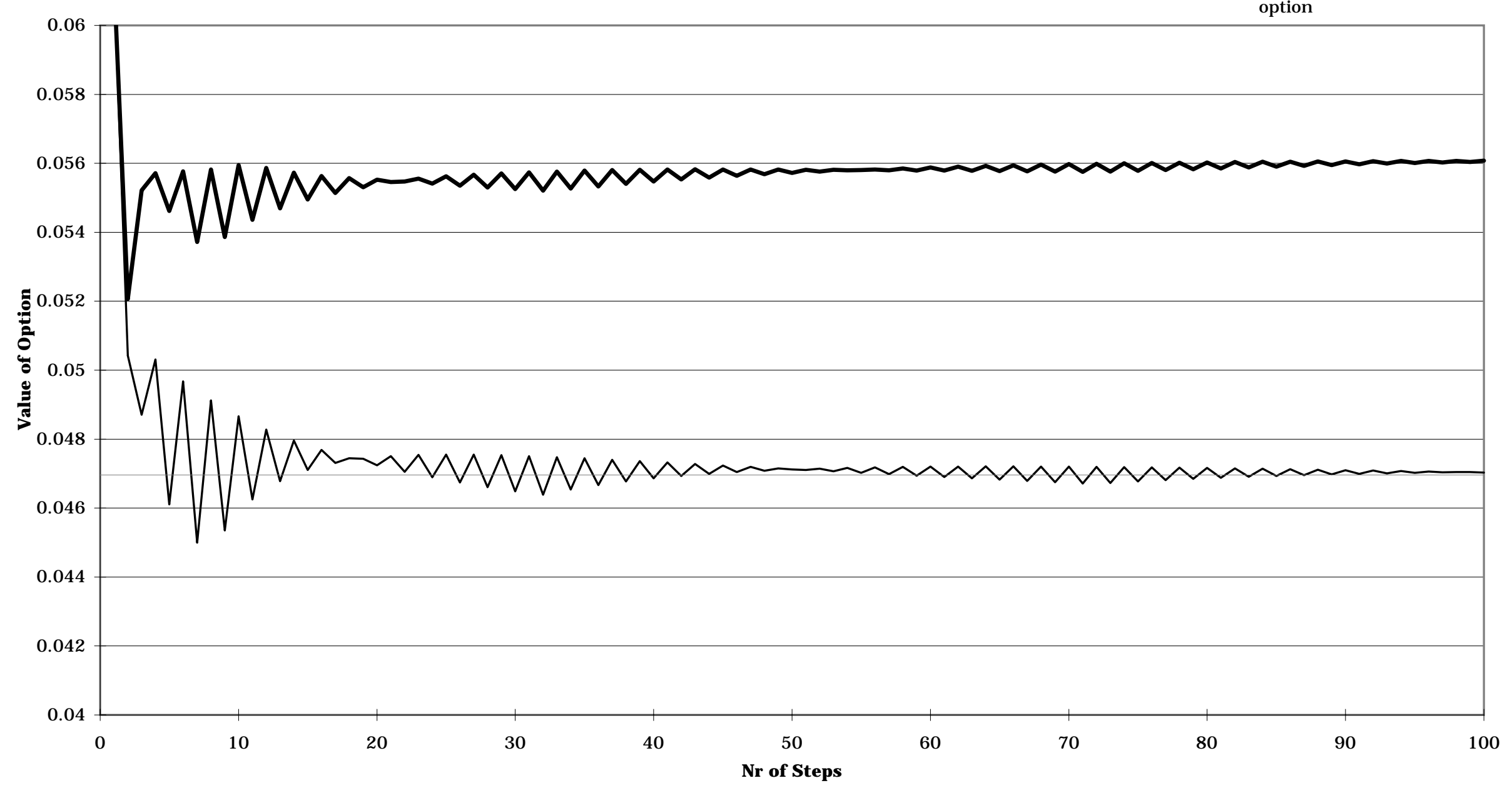




\section{Figure 1b: Value of Put Option}

$\mathrm{S}=1, \mathrm{~K}=1, \mathrm{R}=0.07$ (flat term structure),

$\sigma 1=0.20, \sigma 2=0.01, \rho(r, S)=0, a=0.1$

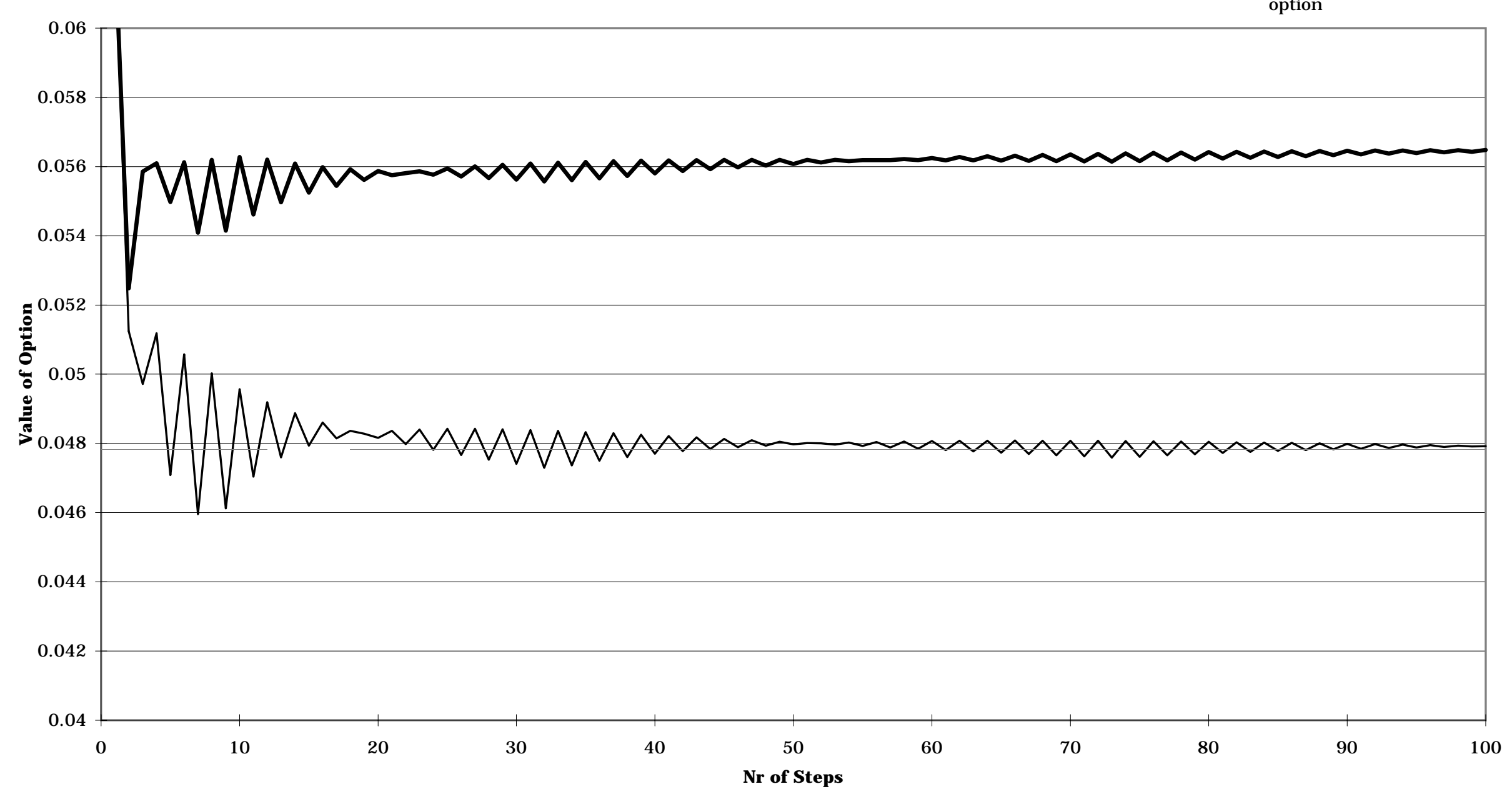

European option value, forward rate adjusted tree American option value. orward rate adjusted tree Analytic value European Analytion 


\section{Figure 1c: Value of Put Option}

$\mathrm{S}=1, \mathrm{~K}=1, \mathrm{R}=0.07$ (flat term structure),

$\sigma 1=0.20, \sigma 2=0.01, \rho(\mathrm{r}, \mathrm{S})=0.5, \mathrm{a}=0.1$

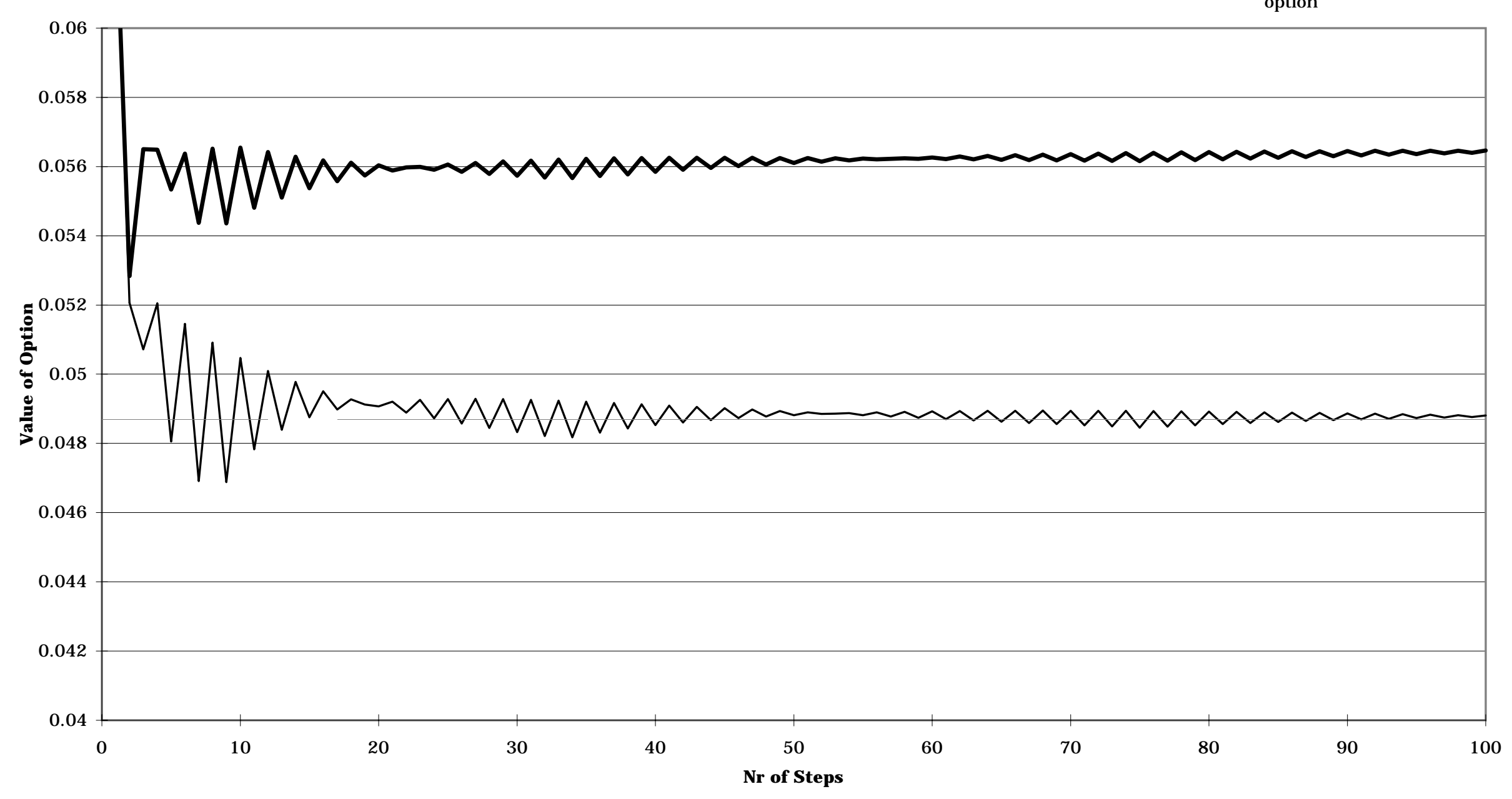

European option value, forward rate adjusted tree

forward rate adjusted tree

Analytic value European

option
American option value. 
Figure 1d: Value of Put Option

$\mathrm{S}=1, \mathrm{~K}=1, \mathrm{R}=0.07$ (flat term structure),

$\sigma 1=0.40, \sigma 2=0.01, \rho(r, S)=0, a=0.1$

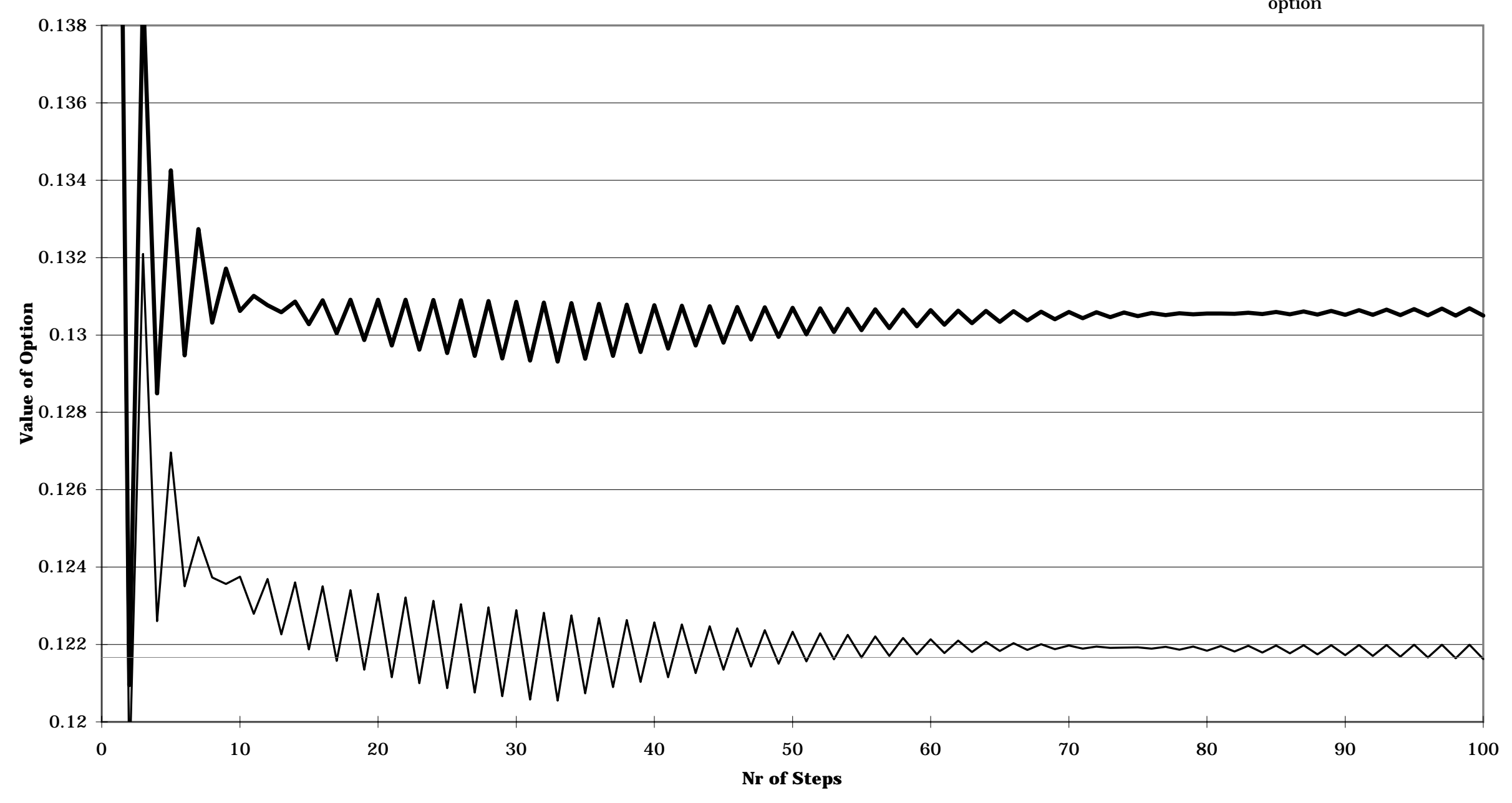
forward rate adjusted tree

American option value.

forward rate adjusted tree

Analytic value European

option

European option value, 
Table 1: The Influence of Stochastic interest rates

$\mathrm{S}=1, \mathrm{~K}=1, \sigma 2=0.01, \mathrm{a}=0.1$, steps $=100$

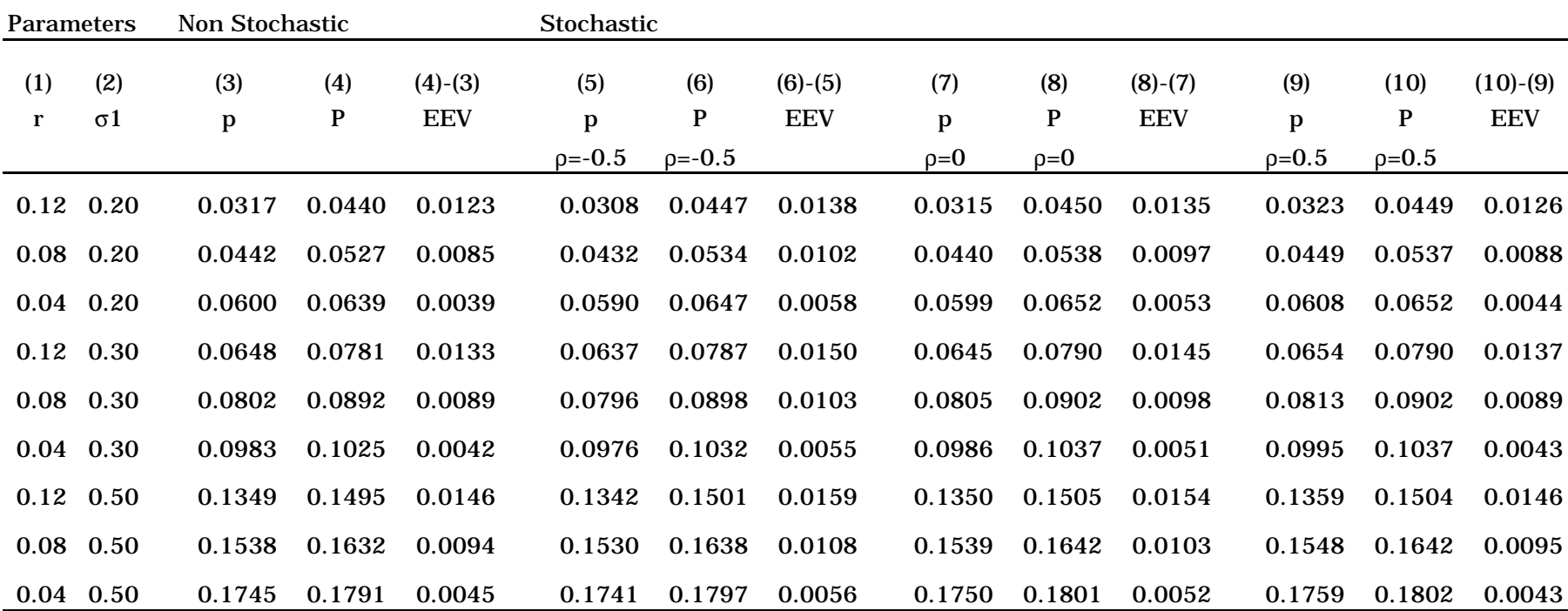

Table 1 shows the difference between American put option prices with and without stochastic interest rates. In case of stochastic rates the correlation between the underlying stock process and interest rate process is allowed to vary.

Columns (1) and (2) represent the parameter input for $r$, the continuously compounded risk-free rate, and $\sigma 1$, the volatility of the underlying asset. The term structure of the interest rate process is flat. The strike price and the initial stock price are equal to 1 . The time to maturity of the option is 1 year. Column (3) shows the European put option value, $p$, in case the interest rate process is non stochastic (i.e. it is fixed at rate r). Column (4) shows the American put option values, $P$, in case the interest rate process is non stochastic. The next column shows the difference between $\mathrm{P}$ and $\mathrm{p}$ and should be interpreted as the early exercise value, eev, of the American option. Columns (5) to (10) show values similar to columns (3) and (4) under a stochastic interest rate process. The volatility, $\sigma 2$, of the stochastic interest rate process is 0.01 . The mean reversion rate, a, is equal to 0.1 . The correlation between the interest rate process and the underlying stock price process for the columns (5) and (6), (7) and (8), (9) and (10) is respectively -0.5, O and 0.5. The early exercise values are shown as differences between $\mathrm{P}$ and $\mathrm{p}$. 
Figure 2.1: The Influence of $\sigma 1$ on the relation between $\rho(r, S)$ and the American Option Value

$\mathrm{S}=1, \mathrm{~K}=1, \mathrm{R}=0.08$ (flat term structure), $\sigma 2=0.01, \mathrm{a}=0.1$, steps $=100$

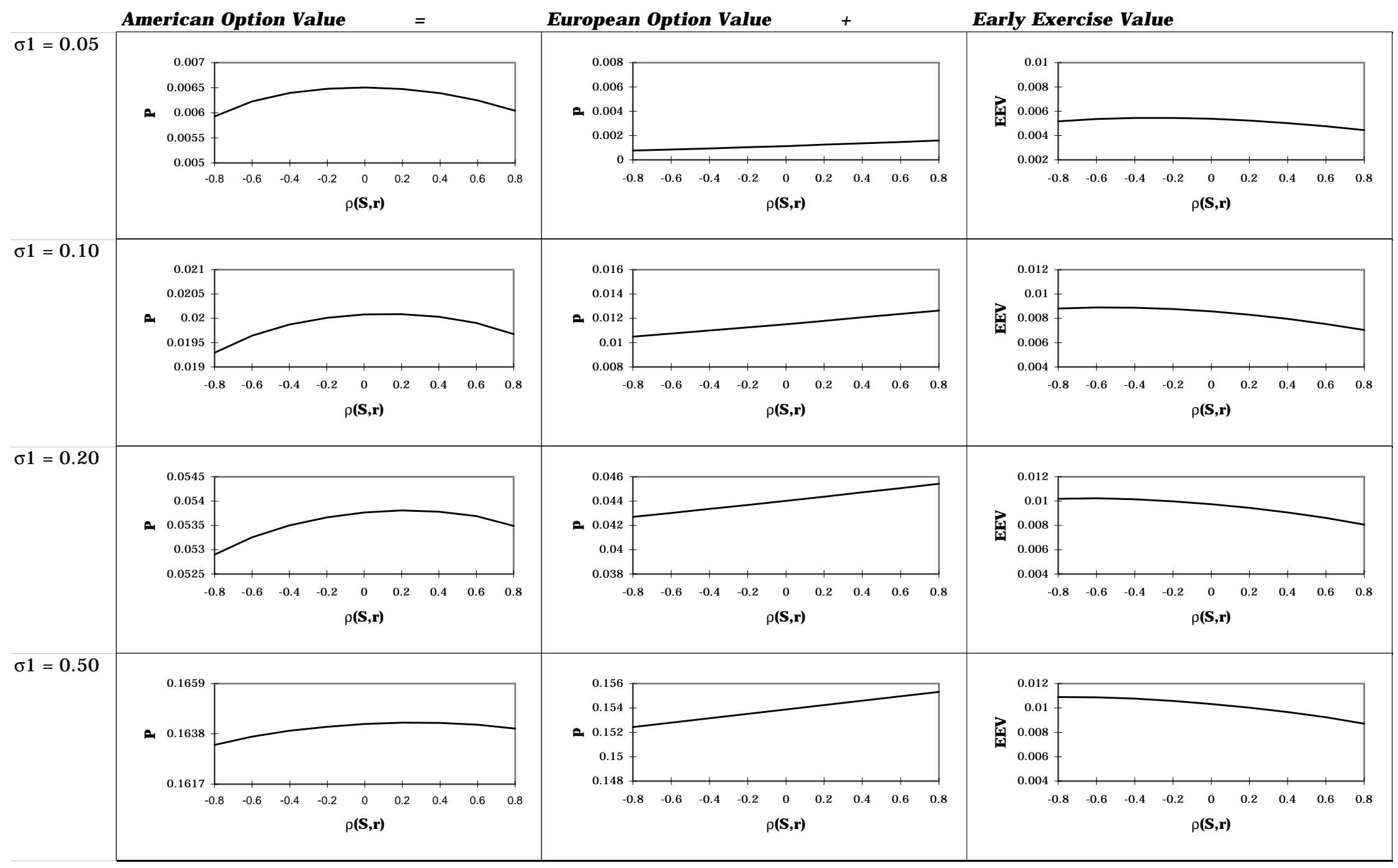


Figure 2.2: The Influence of $r$ on the relation between $r(r, S)$ and the American Option Value

$\mathrm{S}=1, \mathrm{~K}=1, \sigma 1=0.20, \sigma 2=0.01, \mathrm{a}=0.1$, steps $=100$

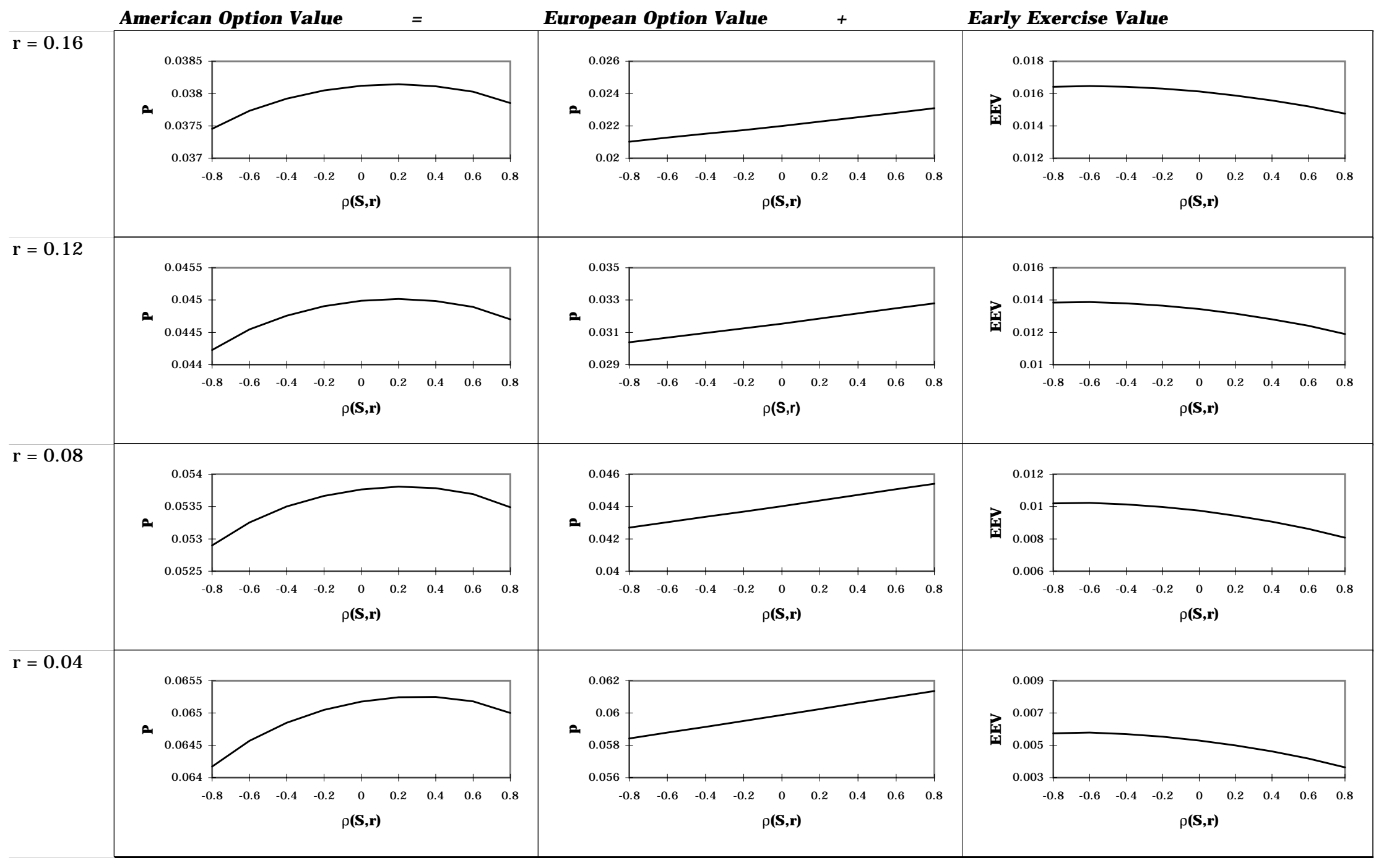


Table 2: Comparison with others

$\mathrm{S}=1, \mathrm{~K}=1, \sigma 2=0.0595, \rho(\mathrm{r}, \mathrm{S})=-0.3, \mathrm{a}=0.1$, steps $=100$

\begin{tabular}{|c|c|c|c|c|c|c|c|c|c|c|c|c|c|}
\hline \multicolumn{2}{|c|}{ Parameters } & \multicolumn{2}{|c|}{ Non Stochastic } & \multirow{3}{*}{$\begin{array}{c}(5) \\
\mathrm{P}^{\mathrm{H} 20}{ }_{\mathrm{NSR}} \\
\end{array}$} & \multirow{3}{*}{$\begin{array}{c}(5) \\
\mathrm{P}^{\mathrm{H} 200}{ }_{\mathrm{NSR}} \\
\end{array}$} & \multirow{3}{*}{$\begin{array}{c}(6) \\
\mathrm{P}^{\mathrm{C} 100}{ }_{\text {NSR }}\end{array}$} & \multirow{3}{*}{$\begin{array}{c}(7) \\
\mathrm{P}^{\mathrm{MV} 100}{ }_{\text {NSR }} \\
\end{array}$} & \multicolumn{2}{|l|}{ Stochastic } & \multirow{3}{*}{$\begin{array}{c}(10) \\
\mathrm{P}_{\mathrm{SR}}^{\mathrm{MV} 2} \\
\end{array}$} & \multirow{3}{*}{$\frac{(10)-(8)}{(8)}$} & \multirow{3}{*}{$\begin{array}{c}(11) \\
\mathrm{P}^{\mathrm{MV} 100}{ }_{\mathrm{SR}}\end{array}$} & \multirow{3}{*}{$\frac{(11)-(8)}{(8)}$} \\
\hline (1) & (2) & (3) & $\begin{array}{c}\text { (4) } \\
\text { G.J }\end{array}$ & & & & & $\begin{array}{r}\text { (8) } \\
\text { H200 }\end{array}$ & $\begin{array}{l}\text { (9) } \\
\text { C }\end{array}$ & & & & \\
\hline $\mathrm{r}$ & $\sigma 1$ & $\mathrm{p}$ & $\mathrm{P}_{\text {NSR }}^{\mathrm{GJ}}$ & & & & & $\mathrm{P}^{\mathrm{H} 200}{ }_{\mathrm{SR}}$ & $\mathrm{P}_{\mathrm{SR}}^{\mathrm{C}}$ & & & & \\
\hline 0.125 & 0.5 & 0.1327 & 0.1476 & 0.1490 & 0.1489 & 0.1489 & 0.1480 & 0.1509 & 0.1510 & 0.1526 & $1.1 \%$ & 0.1611 & $6.7 \%$ \\
\hline 0.080 & 0.4 & 1170 & 0.1258 & 0.1260 & 0.1262 & 0.1262 & 0.1258 & 0.1283 & 0.1284 & 0.1296 & $1.0 \%$ & 0.1392 & $8.5^{\circ}$ \\
\hline 0.045 & 0.3 & 0.0959 & 0.1005 & 0.0994 & 0.1002 & 0.1002 & 0.1006 & 0.1026 & 0.1026 & 0.1041 & $1.5 \%$ & 1144 & $11.5 \%$ \\
\hline 0.020 & 0.2 & 0 & 0.0712 & 0.0705 & 0.0707 & 0.0708 & 0.0713 & 0.0733 & 0.0734 & 0.0749 & $2.2 \%$ & 0.0859 & $17.2 \%$ \\
\hline 0.005 & 0.1 & 3 & 0.0377 & 0.0373 & 0.0375 & 0.0375 & 377 & 404 & 0.0404 & 0.0415 & $2.8 \%$ & 0.0551 & $36.3 \%$ \\
\hline 0.090 & 0.3 & 0.0761 & 0 & 0 & 8 & 0 & 0 & 87 & 0. & 2 & $1.7 \%$ & 00 & 2 \\
\hline 0.0 & 0.2 & 0.0600 & 0 & 0 & 0 & 0 & 0 & 0.0664 & 0 & 5 & $\%$ & 89 & 18 \\
\hline 0.010 & 0.1 & 349 & 0.0357 & 0.0356 & 0.0356 & 0.0356 & 0.0358 & 0.0384 & 0.0384 & 0.0396 & $3.0 \%$ & 0.0533 & $38.7 \%$ \\
\hline 0.080 & 0.2 & 0.0442 & 0.0525 & 0.0536 & 0.0532 & 0.0533 & 0.0527 & 0.0551 & 0.0551 & 0.0561 & $1.8 \%$ & 0.0672 & $22.0 \%$ \\
\hline 0.020 & 0.1 & 0.0304 & 0.0322 & 0.0324 & 0.0322 & 0.0322 & 0.0322 & 0.0349 & 0.0349 & 0.0357 & $2.4 \%$ & 0.0498 & $42.7 \%$ \\
\hline 0.120 & 0.2 & 317 & 0.0439 & 0.0452 & 0.0445 & 0.0446 & 0.0440 & 0.0459 & 0.0459 & 0.0468 & $2.0 \%$ & 0.0577 & $25.8 \%$ \\
\hline 0.030 & 0.1 & 0.0263 & 0.0292 & 0.0295 & 0.0294 & 0.0294 & 0.0293 & 0.0317 & 0.0317 & 0.0326 & $2.7 \%$ & 0.0467 & 47.3 \\
\hline
\end{tabular}

Table 2 shows the difference between American put option prices with and without stochastic interest rates using our approach in comparison to Geske and Johnson (1984), Ho, Stapleton and Subrahmanyan (1997) and Chung (1997). Underneath they will be denoted by GJ, HSS and C respectively.

Columns (1) to (3) are from GJ 's Table 1. Columns (1) and (2) represent the parameter input for $r$, the continuously compounded risk-free rate, and $\sigma 1$, the volatility of the underlying asset. The term structure of the interest rate process is flat. The strike price and the initial stock price are equal to 1 . The time to maturity of the option is 1 year. Column (3) shows the European put option value, p, in case the interest rate process is non stochastic (i.e. it is fixed at rate r). Columns (4), (5), (6) and (7) show the American put option prices in case interest rates are non stochastic* using GJ's approach, HSS's approach with 200 binomial steps, C's approach with 200 steps and our approach with 100 steps.

Columns (8) and (9) show the American put option prices in case interest rates are stochastic using HSS's approach with 200 steps and C's approach with 200 steps respectively. These two columns were taken from C's Table 1 . In this case volatilities are 2 percent for bonds with a maturity of $1 / 2$ year and the coefficient of correlation between the log stock price and the log zero-coupon bond price is 0.3.

Columns (10) and (11) show option values using our approach. To arrive at a bond price volatility of 2 percent, we set the parameters $\sigma 2$ to 0.0595 and a to 0.1 . The volatility of the underlying asset, $\sigma 1$, was adjusted, in order to make the European put option price under stochastic interest rates equal the non stochastic equivalent. Like in HSS and $\mathrm{C}$ this was done to make stochastic and non stochastic results comparable. Column (10) shows the American put option value using Richardson extrapolation. This technique is similar to HSS and C. The American put option value is estimated by twice the value of an American option where early exercise is only allowed at $t=1 / 2$ minus the European option value. Column (11) shows the results using our approach in a tree with 100 steps.

*: For HSS and $\mathrm{C}$ non stochastic means setting the volatility of the bond at $\mathrm{t}=1 / 2$ to 0.0002 percent and the correlation coefficient to $O$. In our approach we consider the interest rate deterministic. However, taking the parameter settings the same as HSS and C does not change our results. 
Table 3: The Influence of the shape of the term structure

$\mathrm{K}=1, \mathrm{~S}=1, \sigma 2=0.01, \mathrm{a}=0.1$, steps $=100$

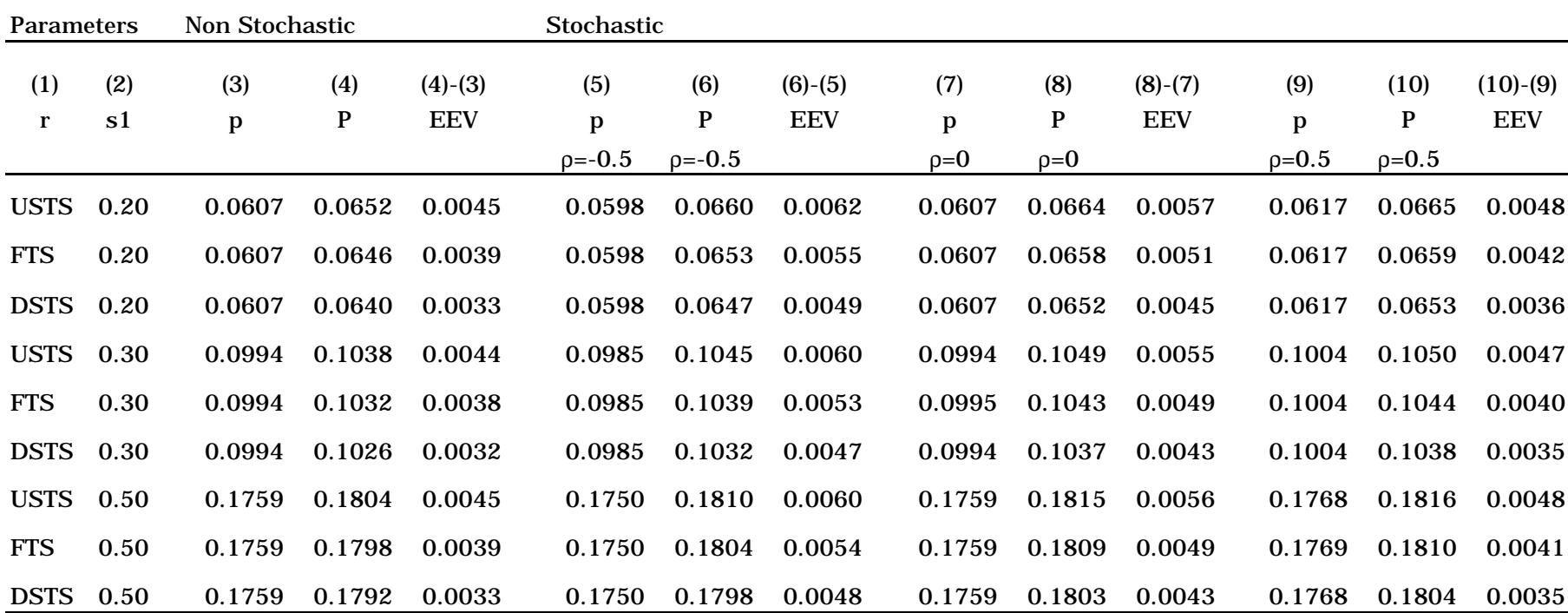

Table 3 shows the difference between American put option prices with and without stochastic interest rates. In case of stochastic rates the term structure of the underlying interest rate process is allowed to vary.

Columns (1) represents the parameter input for $r$, the continuously compounded risk-free rate. The term structure can take three shapes:

(a) an upward sloping term structure, USTS, with $r(t)=0.08-0.05 \exp (-0.18 t)$,

(b) a flat term structure, FTS, with $r(t)=0.0382$ and

(c) a downward sloping term structure, DSTS, with $\mathrm{r}(\mathrm{t})=-0.00353+0.05 \exp (-0.18 \mathrm{t})$.

Underneath this text these three term structures are represented in a graph.

Column (2) represents the volatility of the underlying asset, $\sigma 1$. The strike price and the initial stock price are equal to 1 . The time to maturity of the option is 1 year. Column (3) shows the European put option value, p, in case the interest rate process is non stochastic (i.e. it is fixed at rate r). Column (4) shows the American put option values, $\mathrm{P}$, in case the interest rate process is non stochastic. The next column shows the difference between $\mathrm{P}$ and $\mathrm{p}$ and should be interpreted as the early exercise value, eev, of the American option. Columns (5) to (10) show values similar to columns (3) and (4) under a stochastic interest rate process. The volatility, $\sigma 2$, of the stochastic interest rate process is 0.01 . The mean reversion rate, $a$, is equal to 0.1 . The correlation between the interest rate process and the underlying stock price process for the columns (5) and (6), (7) and (8), (9) and (10) is respectively -0.5, $\mathrm{O}$ and 0.5 . The early exercise values are shown as differences between $\mathrm{P}$ and $\mathrm{p}$.

Shape of the term structures

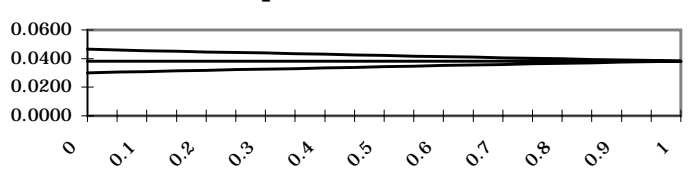

